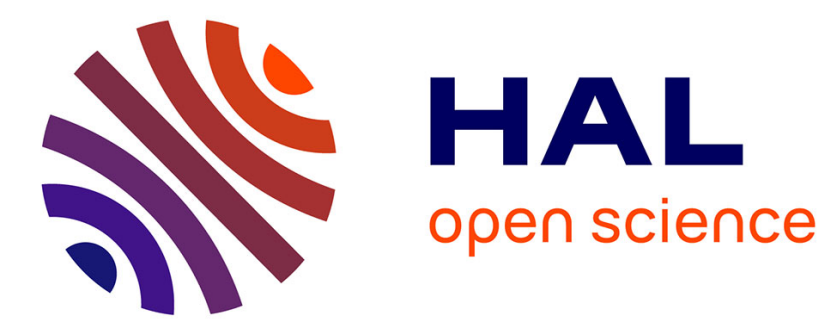

\title{
L'architecture de la sociologie
}

Alban Bouvier

\section{- To cite this version:}

Alban Bouvier. L'architecture de la sociologie. Revue du MAUSS, 2006, 28, pp.391 - 402. ijn_01081435

\section{HAL Id: ijn_01081435 \\ https://hal.science/ijn_01081435}

Submitted on 7 Nov 2014

HAL is a multi-disciplinary open access archive for the deposit and dissemination of scientific research documents, whether they are published or not. The documents may come from teaching and research institutions in France or abroad, or from public or private research centers.
L'archive ouverte pluridisciplinaire HAL, est destinée au dépôt et à la diffusion de documents scientifiques de niveau recherche, publiés ou non, émanant des établissements d'enseignement et de recherche français ou étrangers, des laboratoires publics ou privés. 
<paru dans Revue du MAUSS, $n^{\circ} 28,2006$, pp.391-402>

\title{
L'ARCHITECTURE DE LA SOCIOLOGIE
}

\author{
par Alban Bouvier, \\ Université de Provence
}

Ma thèse est que le principal manque de la sociologie contemporaine n'est pas celui d'une théorie générale unifiée, même si la construction d'une telle théorie serait souhaitable. Une telle théorie générale présenterait un intérêt évident en permettant notamment de démultiplier la portée empirique de modèles de portée initialement locale ou régionale en les ramenant à des principes plus abstraits et donc plus généraux susceptibles d'inspirer à leur tour d'autres modèles particuliers. Mais cet intérêt serait sans proportion aucune avec celui d'une théorie comme celle de Newton en physique (a fortiori comme la mécanique ultérieure, einsteinienne ou quantique, ou la biologie moléculaire), dans la mesure où le contenu substantiel de ses principes explicatifs, à la différence de ceux de la physique ou de la biologie modernes, ne serait pas très sensiblement différents de celui des principes du sens commun (comme c'est le cas des concepts élémentaires de l'économie: marché, coût/bénéfice, choix rationnel, intérêt personnel, etc.) ou de la sagesse universelle édifiée par les écrivains ou les philosophes «moralistes » et les sociologues qui, comme Montesquieu, Tocqueville ou Simmel, ont prolongé la même tradition (les hommes agissent-ils par intérêt ou par générosité, en vertu d'un certain sens de l'honneur ou par crainte, par envie ou par jalousie, par fidélité ou par servilité, etc. ?) alors que les principes de la physique et de la biologie concernent des inobservables qui sont, par nature, inaccessibles au sens commun. En conséquence, l'effet de nouveauté intellectuelle de la «découverte » de ces principes ne peut guère être aussi spectaculaire que celui de la découverte de la structure de l'atome ou du code génétique. En outre le nombre, fort probablement très étendu, de principes élémentaires nécessaires, comme le suggère le très partiel début d'inventaire qui précède, risque de faire perdre à la théorie sociologique générale le caractère d'élégance propre aux théories économes de principes comme la physique et plutôt la faire ressembler à la chimie.

C'est ainsi pour l'essentiel la forme simplement de plus en plus ramifiée et raffinée (choix rationnel comme choix des moyens adaptés aux fins recherchées ou "préférences », choix rationnel comme cohérence des préférences entre elles, choix rationnel comme cohérence des croyances au fondement des préférences, etc.) que l'on donne à ces principes et la méthodologie parfois sophistiquée (souvent mathématique) que cette mise en forme requiert qui font le véritable intérêt théorique de ces principes (comme le montrent par exemple l'axiomatisation de la théorie de la décision ou même le simple usage de la théorie des graphes en analyse de réseaux) plus que leur contenu proprement substantiel, à la différence des principes des sciences de la nature.

Ce dont la sociologie manque toutefois actuellement, me semble-t-il, plus encore que d'une théorie générale, sur laquelle je reviendrai néanmoins en conclusion, c'est _ outre la continuation de l'inventaire et donc de la ramification et du raffinement des concepts et des principes élémentaires de la mise en évidence constamment réitérée de sa nécessaire structure logique d'ensemble et de la place qu'occupent dans cette structure d'ensemble les diverses recherches sociologiques particulières. Un corollaire de la thèse que je soutiens est que l'opacité fréquente de cette structure et, en conséquence, de la place respective qu'y occupent les diverses recherches particulières est due à un laxisme rhétorique considérable (dont ce n'est pas le lieu ici de rechercher la cause).

Cette structure _ ou cette architecture _ logique, dans sa forme la plus générale, n'est pas propre à la sociologie ni aux sciences sociales dans leur ensemble (la spécificité de celles-ci tenant à la nature des principes substantiels que j'ai évoqués) mais est commune à toutes les sciences empiriques quelle que soit la forme que pourrait prendre ensuite une théorie générale.

En prolongeant la métaphore architecturale, je dirais que la sociologie a, comme n'importe quel édifice scientifique, des objets qui se situent à des étages ou à des niveaux différents, lesquels suscitent de façon légitime des programmes de recherche propres à chacun de ces étages ou niveaux, par nature non seulement nullement incompatibles mais pas même conflictuels, comme le sont au contraire des paradigmes. Une autre part considérable de la tâche à accomplir, comme dans n'importe quelle science empirique, consiste à établir des «passerelles» entre ces niveaux (dans un sens ou dans un autre), ce qui peut donner lieu encore à de nouveaux programmes de recherche spécifiques (ce 
que j'appellerai des "programmes passerelles »), eux-mêmes ni incompatibles entre eux ni même conflictuels et qu'il serait en outre absurde de vouloir substituer les uns aux autres au nom d'une nécessaire «évolution » de la discipline. On peut situer par rapport à ces programmes-passerelles un conflit considéré comme majeur en sociologie, le conflit entre individualisme méthodologique et holisme, conflit presque toujours interprété comme un conflit entre paradigmes incompatibles alors qu'il ne s'agit, tout au plus, à mon sens, que d'une opposition de points de vue ou de perspectives quant à la façon d'envisager certains aspects des «programmes-passerelles », si tant est que l'on donne de l'un et de l'autre une interprétation «charitable» et qui ne s'en tient donc pas aux excès rhétoriques (fréquents) de formulations militantes et à strictement parler impensables. J'étudierai successivement ces divers points mais je commencerai par caractériser ce qui me semble pouvoir être considéré comme des conflits de paradigmes en sciences sociales en un sens non abusif du terme, quoique le sens et la portée de ces conflits soit très sensiblement différents de ce que l'on observe dans les sciences de la nature.

\section{1/ Préalable : qu'est-ce qu'un conflit de paradigmes en sciences sociales?}

L'un des éléments les plus intéressants et les plus féconds du concept kuhnien de paradigme réside, me semble-t-il, dans l'idée qu'un paradigme offre un regard particulier sur les phénomènes et qu'un changement de paradigme correspond donc à une autre manière de "voir » ou éventuellement d' "interpréter » les mêmes phénomènes que le précédent. ${ }^{1}$ Kuhn emprunte (via Hanson) un exemple perceptif à Wittgenstein : dans un dessin fameux (désigné communément sous le nom de «canardlapin »), on peut alternativement voir un canard ou un lapin mais jamais les deux à la fois et le fait de voir l'un plutôt que l'autre ne dépend que partiellement de la volonté, de sorte que l'on a parfois du mal à opérer la conversion du regard appropriée. Kuhn dit que le passage de la théorie ptolémaïque à la théorie copernicienne a nécessité une conversion de ce genre : la terre n'a plus été «vue » comme un astre fixe central, mais comme un «astre errant » autour du soleil tandis que le soleil prenait sa place ; Mars, Vénus, Jupiter n'ont plus été « vues » tourner autour de la terre mais autour du soleil ; la lune n'a plus été vue comme une planète parmi les autres errant autour de la terre mais comme le seul astre de ce type tournant autour d'un astre lui-même «errant ». Toutes les observations ont été alors réinterprétées.

Dans cet exemple, un paradigme ou un «type de regard» se substitue toutefois purement et simplement au précédent à la différence de l'exemple visuel de Hanson. Kuhn cite, en revanche, l'exemple des théories de la lumière, plus proche sous ce rapport de l'exemple graphique initial : la lumière peut être vue (plutôt ici : «interprétée ») ou bien comme un paquet de corpuscules ou bien comme une série d'ondes; l'une et l'autre vues (interprétations) se sont alternativement succédées jusqu'à ce qu'on les accepte l'une et l'autre, sous une forme plus sophistiquée. Il me semble que l'on rencontre en sociologie des expériences intellectuelles de ce type (moins le caractère spectaculaire, pour les raisons énoncées plus haut). Ainsi, on peut « voir» le délinquant, sous un certain rapport et jusqu'à un certain point, comme être un peu immature mu par des passions irréfléchies et " commettant des bêtises », mais on peut aussi le « voir », sous un autre rapport et jusqu'à un certain point, comme un «entrepreneur » calculant les coûts probables de ses délits comparés aux avantages escomptés en fonction des risques encourus. ${ }^{2}$ On peut « voir» le prisonnier ou le malade mental, sous un certain rapport et jusqu'à un certain point, comme figés dans des positions fixées par des institutions totalitaires ou bien les «voir», sous un autre rapport et jusqu'à un certain point, comme des «acteurs », voire comme des «metteurs en scène» de leur «rôle » dans l'institution. Certains paradigmes peuvent caractériser les individus, comme ceux qui précèdent, d'autres les relations entre les individus ; on peut ainsi «voir » les relations sociales sous l'angle de l'échange mais aussi sous l'angle du conflit. Un paradigme bien construit développera systématiquement l'une de ces vues ou de ces interprétations, ce qui supposera de circonscrire celles-ci conceptuellement de façon claire et simplifiée, i.e. de les modéliser (ce qu'a tout spécialement fait la théorie du choix rationnel, proposant sous ce rapport l' " interprétation » paradigmatique probablement à ce jour la plus achevée du social) par opposition à ce qui n'est encore qu'une « schématisation » intuitive.

\footnotetext{
${ }^{1}$ Kuhn oscille explicitement entre ces deux versions, l'une très forte, l'autre plus faible

${ }^{2}$ On aurait là une variante (étroite) de la théorie du choix rationnel.
} 


\section{2/ L'analyse de niveau, les programmes de recherche de niveaux et les programmes de recherche « passerelles » entre les niveaux.}

Le jugement de François Dubet, dans le premier volume consacré à la thématique présente, concernant l'éclatement de la sociologie contemporaine et notamment le fait, qui semble effectivement incontestable, qu'un grand nombre d'études relèvent actuellement d'une approche interactionniste constitue un bon point de départ quant à la reconnaissance de l'existence nécessaire de différents niveaux d'analyse ainsi que de passerelles entre ces niveaux. Dubet note le « véritable 'triomphe pratique' » de l'interactionnisme (p. 227), triomphe qui révèle, dit-il avec une certaine ironie, une véritable "passion pour le 'micro'» (id.). Il est bien clair que ces études se situent au niveau microsociologique en tant que tel et même, plus précisément, au plus bas niveau de l'analyse sociologique, celui des individus (et de leurs interactions).

Dubet ajoute que l'on s'éloigne ainsi des préoccupations de la sociologie classique (en entendant par là essentiellement la tradition dukheimienne). Et il précise ainsi son jugement : «l'on s'interrogera longtemps pour savoir comment on passe des interactions individuelles à des faits collectifs » (p. 227) et prend deux exemples : 1: «il est utile d'étudier les interactions dans la classe, mais on saute malaisément de ces interactions aux régularités statistiques enregistrées sur des cohortes d'élèves »; $2:$ "On comprend bien comment on devient fumeur de marijuana sans expliquer pour autant pourquoi une 'société' fume plus ou moins» (p. 228). C'est en partie à peu près ce que dit Caillé lorsqu'il écrit que « depuis une trentaine d'années, l'évolution dominante de la discipline s'est caractérisée par le passage d'une perspective macrosociologique à une optique microsociologique, inspirée par l'interactionnisme symbolique (aujourd'hui relayée par l'analyse de réseaux (...)» (p. 269). On pourrait dire, en prenant l'exemple de l'évolution comparable de la sociologie de l'éducation, que, dans les années 60-70, les innovations théoriques étaient essentiellement au niveau macrosociologique et l'étude de ce niveau donnait lieu à des programmes de recherche spécifiques. Ainsi, c'est au niveau macrosociologique que se situe l'essentiel de l'effort méthodologique de Raymond Boudon dans l'Inégalité des chances (comme celui de James Coleman ou celui de Pierre Bourdieu à la même époque), et dans la préface à la première édition, c'est bien sur ce niveau que Boudon attirait aussi l'attention. Boudon construisait des modèles de simulation de sociétés (française et américaine, en l'occurrence) quant à leur stratification et leur mobilité sociales, modèles dont le but était de combler les lacunes des données empiriques effectives. Et c'est d'abord l'usage de ces modèles simulateurs qui marque, à mon sens, l'originalité méthodologique de Boudon la plus incontestable dans ce champ de recherches, vu notamment les conséquences particulièrement non triviales qu'il tirait de la construction de ces modèles. Par ailleurs, Boudon recherchait les fondements microsociologiques de ces structures macrosociologiques et il modélisait en conséquence le comportement des individus. Or, dans la préface à la seconde édition de l'Inégalité des chances, c'est sur la modélisation des fondements micro du macro, i.e. sur un "programme-passerelle », que Boudon insiste, cette fois, vu probablement à la fois l'évolution du contexte théorique et celle de ses propres intérêts méthodologiques. Mais, à elle seule, l'analyse macrosociologique est bien l'objet possible de programmes propres (et Boudon en développait précisément un dans L'inégalité des chances). Ce qui est caractéristique de la sociologie de l'éducation anglo-saxonne des années 80_90, c'est, en revanche l'analyse de niveau purement microsociologique. Et il s'agit ici exclusivement un programme d'analyse de niveau, non pas un programme-passerelle ou un programme qui serait prolongé par un programme-passerelle (une analyse des fondements micro du macro).

L' «évolution» dont parlent Caillé et Dubet ne requiert ici aucune «conversion du regard », aucune manière différente de «voir » les choses (à la façon du ciel que Copernic donne à «voir », comparé au ciel de Ptolémée ou des deux théories rivales de la lumière) et ne constitue donc nullement une « révolution » ni même une micro-révolution (comme Kuhn en repère également) paradigmatique, comme les sociologues de l'éducation anglo-saxons ont pourtant parfois présenté leur entreprise. Un changement de niveau d'analyse comparable en physique serait, par exemple, le passage d'une analyse des relations entre, d'un côté, le volume et la pression d'un gaz, de l'autre la chaleur de ce gaz (ou réciproquement) _ niveau auquel se situe la fameuse loi de Mariotte _à une analyse des interactions entre les molécules de ce gaz. Dans cet exemple, il s'agit bien de descendre du niveau macro au niveau micro pour expliquer les phénomènes macro (modification de volume et de pression, modification de 
température) comme des effets de phénomènes micro (les mouvement moléculaires), sans qu'il y ait là du tout renversement d'un paradigme par un autre. En revanche, ces programmes d'analyse de niveaux ont parfois connu, en outre, des révolutions paradigmatiques internes à leur niveau, telle l'analyse microphysique, qui a connu un bond grâce au paradigme de la mécanique quantique.

A strictement parler, le "passage » de l'interactionnisme symbolique (ou dramaturgique) à l'analyse de réseaux qu'évoque Caillé (p. 269) ne relève pas non plus d'un changement de paradigme : il s'agit bien d'envisager les mêmes objets (donc a fortiori à un même niveau, en l'occurrence microsociologique) mais pas encore sous deux angles ou avec deux regards différents : l'analyse de réseau s'intéresse en effet à des conditions plus élémentaires des interactions que l'interactionnisme dit symbolique : elle s'intéresse à des conditions de possibilité même des interactions : pour qu'il y ait des interactions, il faut qu'il y ait des relations, que l'analyse de réseau donne les moyens de décrire et de modéliser. Que l'interactionnisme dramaturgique ait historiquement précédé l'analyse de réseaux alors qu'il lui succède logiquement n'est qu'une curiosité de l'histoire des idées.

La dépréciation relative actuelle du niveau d'analyse macrosociologique ou le désintérêt relatif à son égard ne peuvent donc relever que d'une sorte de dérive que décrit fort bien Dubet parce qu'il se réfère implicitement à la structure d'ensemble de la sociologie que je m'efforce ici d'expliciter. La source n'en est pas du tout ici l'absence d'une théorie générale substantielle (la méconnaissance de principes substantiels élémentaires ou le défaut de leur articulation) mais réside dans un réquisit à la fois plus banal et plus élémentaire : l'exposition réitérée de l'architecture logique d'une discipline par nature « multi-niveaux ».

Toutefois, présenter à son tour, comme le font certains démographes (Daniel Courgeau), l'analyse «multi-niveaux » elle-même comme un nouveau paradigme relèverait, à mon sens, d'une confusion analogue à celle signalée à propos de la sociologie de l'éducation. Cependant, quand on parle l'analyse «multi-niveaux », on évoque, en outre et à juste titre, l'idée qu'il y a d'autres niveaux que le micro et le macro au sens habituel de ces termes. Ces derniers termes sont cependant à prendre en un sens relatif car on peut envisager des effets de "globalité » différents à différents niveaux intermédiaires : les interactions entre des individus peuvent avoir des effets au niveau d'un groupe (association, parti, église), qui constitueront dès lors du «macro » par rapport aux individus mais du « micro » par rapport à société dans son ensemble ; si l'on ne distingue que trois niveaux, on peut commodément appeler «méso » le niveau intermédiaire (l'exigence restant de revenir à un moment ou un autre au niveau des individus). On ne confondra pas davantage avec une théorie générale le raffinement de l'analyse multi-niveaux que constitue le «systémisme» (Mario Bunge), qui ajoute l'idée qu'il y a des effets probables de feed-back du macrosociologique sur le microsociologique (par exemple, le fait d'être membre d'un parti ou d'une église ou d'habiter un pays démocratique ou au contraire totalitaire ou de vivre dans un pays dans lequel le taux de chômage est élevé influe sur le comportement). Mais il n'y a là nul changement de «paradigmes » à envisager un niveau plutôt que l'autre ou les effets de l'un sur l'autre.

\section{3 / Les programmes de recherche «passerelles » symétriques.}

La dénomination d'analyse multi-niveaux a l'inconvénient, par ailleurs, de ne pas suggérer, en tant que telle, l'exigence de programmes passerelles. Or, c'est bien sur la nécessité de ces programmes que, dans un autre langage, Dubet insiste, plus encore que sur le discrédit de l'analyse de niveau macrosociologique. Dans un langage à peine différent du sien, on pourrait dire que ce qui fait défaut du point de vue de Dubet dans le champ global de la sociologie, vu la prédominance des études interactionnistes, c'est surtout l'étude du passage du micro au macro et, plus précisément, l'étude des effets au niveau macro de comportements observés au niveau micro.

Il convient, bien entendu, d'ajouter à ce que dit Dubet que 1'on peut a priori parcourir la relation causale entre le micro et macro dans les deux sens _ on pourrait alors parler commodément d'analyse progressive pour désigner ce que Dubet a en vue (Hayek aurait parlé d'analyse «compositive» ou «synthétique ») mais on peut aussi chercher les fondements (c'est-à-dire les causes) micro des phénomènes observés au niveau macro (à titre d'effets)_ et parler alors d'analyse régressive (la méthode "analytique » de Hayek). C'est celle que pratiquait Boudon dans L'inégalité des chances. En principe, bien entendu, les deux types de démarche devraient se rejoindre. 
Mais, de façon générale, Boudon _ pour prolonger les références à l'un des auteurs du premier volume les plus réputés sur le sujet, et discuté en bonne place par Alain Caillé _ expose une version particulière de la recherche des fondements micro du macro, non seulement parce qu'il envisage l'idée d'un programme-passerelle entre macro et micro presque exclusivement dans le sens « régressif » et non dans le sens «progressif» (qui était, parmi les classiques, celui auquel se consacrait plutôt Simmel), ni non plus parce qu'il envisage le programme «régressif» comme une simple reconstruction d'un individu idéal-typique anonyme et non comme une remontée à des individus historiques effectifs (comme le Weber de l'Ethique protestante) mais encore parce que, fidèle en fait à la modélisation «autrichienne» particulière (de Menger à Hayek et von Mises), il s'intéresse essentiellement aux effets agrégés (complexes) non voulus. Il y a de très bonnes raisons à cela parce que ces effets, étant paradoxaux (i.e. contraires à ce qui à quoi les acteurs s'attendent), présentent un intérêt intellectuel plus évident. Mais cette insistance peut finir par voiler bien d'autres choses. Ainsi le schéma boudonien (ou mengerien ou hayekien) n'intègre pas du tout les phénomènes macro qui sont les effets réussis d'intentions collectives; Weber (qui s'intéresse à des effets agrégés simples), non plus, au demeurant ${ }^{3}$. C'est pourtant le cas globalement parlant des institutions. Il n'y a aucune raison logique, dans une perspective micro-fondationnelle, pour ne pas s'y intéresser. Boudon, pas plus que Weber, ne s'intéresse non plus beaucoup aux groupes intermédiaires (le niveau méso). ${ }^{4}$ Cette absence d'analyse des institutions et des groupes conduit a fortiori à ne pas s'intéresser aux effets de feed-back (feed-back des phénomènes voulus _ les institution _ou des phénomènes non voulus sur les individus). Mais il n'y a toujours pas là des manières de «voir» le social différentes, donc d'autres «paradigmes »; simplement des façons plus ou moins complètes de remplir les programmespasserelles.

\section{4/ Le conflit de perspectives IM / holisme.}

On peut aborder maintenant de front la question : qu'est-ce que spécifiquement que l'IM? Sa spécificité, aussi bien chez Weber que chez Menger, c'est de s'attaquer aux concepts collectifs, dans la mesure où ils peuvent cacher des entités collectives en quelque sorte "fantomatiques », i.e. dont on sera incapable de dire clairement à quoi elles renvoient empiriquement, sachant que les entités collectives réelles doivent forcément, d'une manière ou d'une autre, être le produit d'individus. Les propositions macrosociologiques peuvent en comporter et la recherche systématique des fondements micro du macro permettra de les éliminer. Le principe de l'IM est donc différent dans son inspiration et dans ses objectifs (éviter les causes fantômes) du principe de l'analyse des fondements micro du macro, quoique le premier appelle le second comme son instrument. Aussi y a-t-il un sens à adopter le principe de l'IM même quand le but reste la connaissance du seul niveau macro (comme c'est le cas en démographie). On notera que quand l'on est, symétriquement, au seul niveau micro lui-même, des concepts collectifs peuvent fort bien se mêler aux analyses, de sorte que la vigilance propre à l'IM reste de mise; mais les risques sont évidemment bien moins considérables qu'au niveau macrosociologique.

Dans les faits, c'est surtout au niveau que l'on appelle parfois « méso » (le niveau notamment des groupes) que la question se pose le plus. Cette question de la nature des groupes conduit à celle de la légitimité du holisme (i.e. l'idée qu'un tout social est autre chose que la simple somme ou agrégation de ses parties). Alain Caillé écrit : «la résolution de la dichotomie du holisme et de l'individualisme méthodologiques est la condition sine qua non, l'étape première, d'une sociologie générale enfin recevable», p. 10-1. J'ai cherché à montrer qu'il y avait encore des questions préalables, mais celle-ci est assurément une question capitale. Ce n'est pas que Boudon, Coleman ou Elster rejettent vraiment l'idée qu'il y a une spécificité des groupes ; c'est plutôt qu'ils n'en parlent pratiquement pas. Et lorsque Boudon, par exemple, évoque directement le holisme, c'est, à mon sens,

${ }^{3}$ Hirschman, dans Les Passions et les intérêts, prendra sur ce point délibérément le contre-pied de Weber, dans un style de recherche (descriptif et non reconstructif) des fondements micro du macro néanmoins analogue à celui du Weber de l'Ethique protestante.

${ }^{4}$ Rien sur les Eglises ou les sectes protestantes comme institutions dans L'Ethique protestante; rien non plus sur les communautés religieuses (protestantes, méthodistes, baptistes, catholiques, etc) comme telles, même si le principe de leur étude sera inscrit dans Economie et société. 
tantôt pour le confondre avec celui du déterminisme (par exemple dans son introduction au Traité de sociologie), tantôt en se contentant de formules équivoques qui ne le distinguent pas nettement de la thèse des effets de feed-back du macro ou du «méso» sur le micro. ${ }^{5}$ Cette identification malencontreuse et illégitime _ est certes compréhensible dans le contexte français, où certains des représentants les plus typiques du holisme contemporain (comme Pierre Bourdieu) ont parfois présenté celui-ci dans un cadre déterministe et se sont en outre focalisés sur le rôle des institutions, voire sur le rôle de la structure de celles-ci (en usant, au demeurant, de formules dont on ne peut pas croire qu'elles n'étaient pas outrageusement rhétoriques puisqu'elles tendaient à faire de celles-ci les véritables acteurs du social _ ce qui est proprement impensable). Mais le déterminisme, en tant que tel, est logiquement indépendant du holisme et réciproquement, alors que les effets de feed-back sont, quant à eux, tout à fait intégrables dans une perspective méthodologiquement individualiste.

Au sein même de la tradition méthodologiquement individualiste, Simmel suggère une idée féconde quant à la nature des groupes: c'est que si le sociologue ne doit pas assumer l'existence d'entités collectives spécifiques (i.e. qui ne seraient pas explicables par des effets d'agrégation voulus ou non voulus), il doit prendre en compte le fait que les individus, eux, ont parfois le sentiment d'appartenir à des groupes qui les dépassent et qui seraient des acteurs propres du social, voire les seuls vrais acteurs. Et ce sentiment varie en intensité selon les sociétés. Amartya Sen, un individualiste méthodologique indien aussi sereinement assumé que le sont Coleman ou Boudon, dit ainsi que si l'on demandait à un indien des classes populaires ce qu'il pense de l'état de son bien-être, il ne comprendrait même pas la question ; ou bien il penserait qu'on lui pose la question du bien-être de $s a$ famille car c'est l'entité perçue comme pertinente à presque tous les niveaux de son existence. Rien ne devrait empêcher, dès lors, de partir, dans l'analyse sociologique de ce sentiment qu'ont les individus d'être membres d'un groupe avant d'être des acteurs individuels (à mon sens, c'était là l'intuition intellectuelle profonde de Durkheim, explicite par exemple lorsqu'il parle du sentiment national face au drapeau ou tribal face au totem). Peut-être cela est-il même recommandé dans des sociétés comme l'Inde. Se trouve alors légitimé ce qu'on pourrait éventuellement appeler un holisme méthodologique, qui s'opposerait à l'individualisme méthodologique classique mais seulement en ce que l'ordre dans lequel les problèmes devraient être examinés ne serait pas le même. Encore n'est-il même pas certain qu'on doive partir de ce sentiment subjectif. Mais si l'on n'en part pas, il faudra bien, à un moment où à un autre, dans la remontée du macro vers le micro, tenir compte de l'existence non seulement de ces groupes structurés observables de l'extérieur mais, en outre, du sentiment subjectif (plus ou moins puissant selon les sociétés) qu'ont les individus d'être membres de ces groupes et de l'être parfois avant même que d'être des personnes privées. Il est toutefois, à mon sens, abusif à nouveau de parler ici de conflits de paradigmes (a fortiori incompatibles) car il ne s'agit pas de deux manières différentes de voir ou d'interpréter les phénomènes, seulement de deux manières différentes d'ordonner l'analyse des phénomènes, ce qui légitime en revanche que l'on parle de perspectives différentes.

\section{5/ Un challenge : construire une pluralité de théories générales.}

Revenons pour conclure à l'idée d'une théorie générale substantielle puisque l'utilité en est certaine, même si ce n'est pas la tâche la plus élémentaire et même s'il ne faut pas non plus trop en attendre. Dans son introduction au premier volume consacré à la thématique présente, Alain Caillé écrivait: "La tentative la plus récente d'édifier une sociologie générale, celle que présente James Coleman dans ses Foundations of Social Theory procède d'un individualisme méthodologique intraitable et repose donc sur cet utilitarisme de la théorie de l'action rationnelle (...) dont Parsons avait affirmé au contraire (et à juste titre en l'occurrence) qu'il était précisément ce qui devait être dépassé pour accéder à un point de vue proprement sociologique » (p. 10). Je ne partage pas entièrement le jugement de Caillé sur l'œuvre de Coleman pour diverses raisons. Pour m'en tenir à l'essentiel, je dirais d'abord qu'Alain Caillé construit un «homme de paille» en prêtant à Coleman des idées qui ne sont pas les siennes. Un des traits caractéristiques de la théorie sociale de Coleman est, en effet, qu'il introduit en bonne place le

${ }^{5}$ Cf la définition du holisme dans La logique du social, p. 38-9: « le point de vue selon lequel les structures seraient premières par rapport aux individus et explicatives par rapport à eux ». 
concept de sympathie (en s'inspirant explicitement de Smith) et celui d'identification (en s'inspirant explicitement de Cooley et de G.-H.Mead) pour rendre compte de phénomènes qui échappent irréductiblement, de son propre aveu, à une explication par le self-interest matériel (par exemple le sentiment national ou, plus généralement, celui d'appartenance à un groupe). ${ }^{6} \mathrm{Il}$ y a là déjà assurément beaucoup pour penser «le lien social». On pourrait plus justement reprocher à Coleman à ce sujet, me semble-t-il, de proposer une modélisation de l'identification et de la sympathie encore bien confuse et d'avoir une conception encore bien inchoative de ce qu'est un groupe.

En revanche, et c'est la deuxième chose essentielle, Caillé ne rend pas du tout justice à l'effort analytique de construction d'une théorie générale qu'expriment les Foundations et dont il n'y a aucun équivalent antérieur en sociologie _ Parsons y compris (Peter Blau éventuellement mis à part). Coleman a l'immense mérite de procéder constamment par modélisation conceptuelle et d'articuler de façon serrée modèles sur modèles en intégrant ainsi une partie considérable de la sociologie classique et contemporaine. Il fait certes preuve de trop de précipitation dans l'évaluation des théories respectives, tombe dans le «biais de confirmation » et donc dans le dogmatisme. Il s'arrête en chemin à un nombre de principes explicatifs substantiels extrêmement réduits. Soit ! Mais la voie est ouverte pour construire une théorie plus complexe et plus complète sur cette base. ${ }^{7}$

Le vrai challenge serait de formuler une théorie rivale de celle de Coleman, qui se laisserait guider par un autre « paradigme » et partirait donc d'une autre «base »: non plus un paradigme animé par la «vision» de l'homme rationnel et «intéressé », auquel on doit ajouter pas à pas d'autres caractéristiques (comme la sympathie, à la manière de Coleman, et probablement bien d'autres motivations encore, comme le montre de façon éclatante Jon Elster) mais, par exemple, par la « vision » de l'homme animé par le sens de l'honneur ou du respect qu'on lui doit, auquel on ajouterait pas à pas d'autres caractéristiques, ou bien animé par le sens du don et la générosité, auquel on ajouterait pas à pas d'autres caractéristiques etc. Mais si la tâche visée est bien scientifique, il faudra nécessairement modéliser, donc construire une théorie analytique et systématique comme l'est celle de Coleman. ${ }^{8}$ De telles théories rivales, si elles étaient bien faites et correctement testées empiriquement, ne devraient nullement être incompatibles mais, au contraire, converger.

Des paradigmes aussi différents conserveraient assurément aux diverses recherches sociologiques une allure elle-même très différente. Mais, aucun point de départ ne s'imposant absolument, la diversité de facto des approches en sociologie, prenant leur source première dans des « regards » fort différents, pourrait être ainsi légitimée tout en étant bien mieux articulée et donc bien plus cumulative.

${ }^{6}$ C'est l'objet essentiel du chap. 19 des Foundations (« The Self »).

${ }^{7}$ Une allusion de Coleman à Hirschman (Les passions et les intérêts) montre qu'il n'évacue pas l'idée qu'il y ait une composante historique importante dans la valorisation de la recherche du self-interest matériel, ce qui suggère que, dans d'autres sociétés, ce pourrait être l'honneur qui jouerait le rôle central.

${ }^{8}$ Une entreprise préalable pourrait être de formuler, de dénombrer et d'ordonner les modèles conceptuels implicites dans l' Essai sur le don de Mauss. Ce ne serait déjà pas une mince affaire vu la profusion de l'Essai. 